\title{
Propofol Attenuates Hypoxia/Reoxygenation-Induced Apoptosis and Autophagy in HK-2 Cells by Inhibiting JNK Activation
}

\author{
Huaxin Wang, Xuan Peng, Yayi Huang, Yeda Xiao, Zhuo Wang, and Liying Zhan \\ Department of Anesthesiology, Wuhan University Renmin Hospital, Wuhan, China.
}

\begin{abstract}
Purpose: The aim of this study was to investigate whether propofol could attenuate hypoxia/reoxygenation-induced apoptosis and autophagy in human renal proximal tubular cells (HK-2) by inhibiting JNK activation.

Materials and Methods: HK-2 cells were treated with or without propofol or JNK inhibitor SP600125 for 1 hour and then subjected to 15 hours of hypoxia and 2 hours of reoxygenation (H/R). Cell viability and LDH release were measured with commercial kits. Cell apoptosis was evaluated by flow cytometry. The expressions of p-JNK, cleaved-caspase-3, Bcl-2, and autophagy markers LC3 and 62 were measured by Western blot or immunofluorescence.

Results: HK-2 cells exposed to H/R insult showed higher cell injury (detected by increased LDH release and decreased cell viability), increased cell apoptosis index and expression of cleaved-caspase-3, a decrease in the expression of Bcl-2 accompanied by increased expression of p-JNK and LC3II, and a decrease in expression of p62. All of these alterations were attenuated by propofol treatment. Similar effects were provoked upon treatment with the JNK inhibitor SP600125. Moreover, the protective effects were more obvious with the combination of propofol and SP600125.

Conclusion: These results suggest that propofol could attenuate hypoxia/reoxygenation induced apoptosis and autophagy in HK-2 cells, probably through inhibiting JNK activation.
\end{abstract}

Key Words: Propofol, HK-2 cells, apoptosis, autophagy

\section{INTRODUCTION}

Renal ischemia and reperfusion (I/R) injury is a common problem in clinical surgeries, such as renal transplantation, and prolonged ischemia results in irreversible damage to renal function. ${ }^{1}$ Studies have reported that apoptosis is essential to the pathogenesis of renal injury secondary to I/R. ${ }^{2,3}$ Proximal tubular epithelial cells (PTCs) are particularly vulnerable to hypoxia-induced injury. More importantly, the vital pathogenesis of renal

Received: July 23, 2019 Revised: September 13, 2019

Accepted: September 23, 2019

Corresponding author: Liying Zhan, MD, Department of Anesthesiology, Wuhan University Renmin Hospital, No. 238, Jiefang Road, Wuhan 430060, Hubei, China. Tel: 86-027-88041911-81026, Fax: 86-027-88042292, E-mail: 45123915@qq.com

-The authors have no potential conflicts of interest to disclose.

(C) Copyright: Yonsei University College of Medicine 2019

This is an Open Access article distributed under the terms of the Creative Commons Attribution Non-Commercial License (https://creativecommons.org/licenses/ by-nc/4.0) which permits unrestricted non-commercial use, distribution, and reproduction in any medium, provided the original work is properly cited.
I/R has been widely considered as renal tubular epithelial cells apoptosis. ${ }^{4}$ The close connection between renal tubular epithelial cell apoptosis and renal I/R injury has drawn substantial attention in developing anti-apoptosis therapies.

Propofol (2,6-disopropylphenol), an intravenous sedativehypnotic agent well-known for its utilization in anesthesia, has also been demonstrated to alleviate I/R injury due to its anti-inflammatory and anti-oxidant activities. ${ }^{5}$ However, the precise mechanism thereof remains largely unresolved. Meanwhile, autophagy is an evolutionarily conserved multi-step process that maintains normal function and the structure of the cell and is reported to be involved in renal I/R injury. ${ }^{6}$ Recent research reported that propofol attenuated hypoxia/reoxygenation-triggered neuronal injury via inhibition of autophagy. ${ }^{7}$ However, little is known about the underlying mechanisms by which propofol regulates autophagy.

c-Jun N-terminal kinase (JNK, including JNK 1, 2, 3), also known as stress-activated protein kinase, is another subclass of the mitogen-activated protein kinase signaling pathway in mamma- 
lian cells. JNK is closely related to inflammation, cell apoptosis, and autophagy, ${ }^{8}$ and excessive activation of JNK has been implicated in nephropathy and renal I/R injury. ${ }^{9,10}$ Accordingly, we hypothesized that propofol could attenuate hypoxia/reoxygenation-induced apoptosis and autophagy in human renal proximal tubular cells (HK-2) by inhibiting JNK activation.

\section{MATERIALS AND METHODS}

\section{Cell culture and treatments}

HK-2 cells were purchased from Procell Life Science \& Technology Co., Ltd. (Wuhan, China). The cells were cultured in minimum essential medium supplemented with $10 \%$ fetal bovine serum both from Gibco/Thermo Fisher Scientific (Shanghai, China) and $1 \%$ penicillin and streptomycin under a humidified atmosphere consisting of $5 \% \mathrm{CO}_{2}$ and $95 \%$ air at $37^{\circ} \mathrm{C}$ (control group). Hypoxia and reoxygenation $(\mathrm{H} / \mathrm{R})$ injury was introduced by exposing the cells to hypoxic conditions $\left(1 \% \mathrm{O}_{2}, 5 \% \mathrm{CO}_{2}\right.$ and $94 \% \mathrm{~N}_{2}$ ) for $15 \mathrm{~h}$, followed by reoxygenation under normoxic conditions (reoxygenation) for $2 \mathrm{~h}$ in fresh normal medium (H/R group).

Propofol (Aladdin Reagent Int., Shanghai, China) was dissolved in dimethyl sulfoxide (DMSO) and subsequently diluted in minimum essential medium to final concentrations of 10, 25, 50, and $100 \mu \mathrm{M}$ prior to $\mathrm{H} / \mathrm{R}$ injury (propofol groups); the final concentrations of DMSO were below $0.01 \%$, thereby minimizing its effects. A positive control was set up using cells pretreated with $10 \mu \mathrm{M}$ SP600125 (MedChemExpress, Shanghai, China), which is a specific JNK inhibitor. Finally, a combination of $50 \mu \mathrm{M}$ propofol and $10 \mu \mathrm{M}$ SP600125 was added to the cell cultures at $1 \mathrm{~h}$ before H/R injury to ascertain synergistic effects.

\section{Cell viability analysis}

Cell viability was assessed with Cell Counting Kit-8 from Biosharp (Guangzhou, China). Briefly, after reoxygenation for $2 \mathrm{~h}$, each well was supplemented with $10 \mu \mathrm{L}$ of CCK- 8 solution, followed by $4 \mathrm{~h}$ of incubation at $37^{\circ} \mathrm{C}$. Subsequently, optical density of each well was measured with a microplate reader at a wavelength of $450 \mathrm{~nm}$ (MULTISKAN MK3, Thermo, USA). Higher cell viability leads to higher optical density reading.

\section{Measurement of lactate dehydrogenase by ELISA}

A lactate dehydrogenase (LDH) release assay (Changchun Huili Biotech Co., Ltd, Changchun, China) was used to determine the extent of cell injury by measuring the amount of LDH released into the medium by the cells following the manufacturer's guidance.

\section{Flow cytometry analysis}

Flow cytometry analysis was carried out to assess apoptotic index values in HK-2 cells. Following treatment, HK-2 cells were detached from wells by addition of $0.25 \%$ trypsin, resuspended in phosphate-buffered saline (PBS), and spun down at $1500 \mathrm{rpm}$ for 5 mins. Subsequently, the cells were resuspended in $500 \mu \mathrm{L}$ of binding buffer, followed by incubation with Annexin V-FITC $(5 \mu \mathrm{L})$ and propidium iodide (PI, $5 \mu \mathrm{L})$ for $10-15$ mins at room temperature $\left(20-25^{\circ} \mathrm{C}\right)$ in the dark. Apoptotic cell ratio was detected using a flow cytometer (CytoFLEX, Beckman Coulter Life Sciences, Indianapolis, IN, USA). Early apoptotic cells were differentiated by unique characteristics: positive for Annexin VFITC and negative for PI.

\section{Western blotting}

Cell cultures were harvested and homogenized in ice-cold radioimmunoprecipitation assay buffer with protease inhibitors (Beyotime, Shanghai, China) and incubated for $30 \mathrm{mins}$ at $4^{\circ} \mathrm{C}$. After centrifugation, the supernatants, which contained our target proteins, were transferred to a new vial for storage at $-70^{\circ} \mathrm{C}$ or immediate use. A BCA protein assay kit was then used to quantify the concentrations of proteins (Beyotime, Shanghai, China). The proteins $(40 \mu \mathrm{g})$ were run on $12 \%$ SDS-PAGE gel and then transferred electrophoretically to a polyvinylidene fluoride membrane (Millipore, Shanghai, China). The blots were blocked for $2 \mathrm{~h}$ at $25^{\circ} \mathrm{C}$ with $5 \%$ skim milk in Tris-buffered saline containing $0.1 \%$ Tween 20 (TBST), followed by incubation with primary antibodies, including anti-JNK, anti-p-JNK, anti-caspase-3, antiBcl-2, anti-LC3, anti-p62, and anti- $\beta$-actin antibodies (Proteintech Group, Inc., Wuhan, China) at $4^{\circ} \mathrm{C}$ overnight. Subsequently, membranes were washed with TBST five times for 5 mins each. Then, respective horseradish peroxidase-linked secondary antibodies (Beyotime, Shanghai, China) were added and incubated for $2 \mathrm{~h}$ at $37^{\circ} \mathrm{C}$, followed by washing with TBST. Lastly, ECL solution was applied to the membrane evenly prior to detection of chemiluminescence (Thermo Scientific, Shanghai, China).

\section{Immunofluorescence assay}

The HK- 2 cells were fixed in $4 \%$ paraformaldehyde for 15 mins and washed three times with PBS for 3 mins each time. Then, the samples were treated with $0.5 \%$ Triton X-100 for $20 \mathrm{mins}$, washed, and blocked for $30 \mathrm{mins}$ at $37^{\circ} \mathrm{C}$. Next, the samples were incubated in a wet box at $4^{\circ} \mathrm{C}$ overnight with primary antibodies against LC3 (mouse anti-LC3 antibody or p62 (rabbit antip62 antibody) diluted 100 times in blocking solution. After washing with PBS containing $0.1 \%$ Tween 20 , the cells were incubated with secondary antibodies diluted 100 times (LC3, Cy3-labeled goat anti-mouse IgG; p62, Cy3-labeled goat anti-rabbit IgG) for $1 \mathrm{~h}$ in the dark at $37^{\circ} \mathrm{C}$. Lastly, 4,6-diamidino-2-phenylindole (Beyotime, C1002) was used as a counterstain. After staining, cells were visualized with a fluorescence microscope (BX53, Olympus, Tokyo, Japan).

\section{Statistics analysis}

Statistical analyses were conducted with GraphPad Prism version 6 (GraphPad Software Inc., San Diego, CA, USA). Each experiment was repeated six times. Results are presented as mean \pm 
standard deviation (SD). Statistical significance was analyzed first with one-way analysis of variance and then with Tukey's multiple comparisons test. $p$ values less than 0.05 were considered to represent statistically significant differences.

\section{RESULTS}

\section{Propofol alleviates the reduced cell viability induced by $\mathrm{H} / \mathrm{R}$ injury}

In the present study, we investigated the effect of $\mathrm{H} / \mathrm{R}$ insult on HK-2 cells. As shown in Fig. 1, H/R decreased cell viability by 39\%, compared with the no-insult control group $(p<0.001)$. Propofol significantly alleviated the decrease in cell viability induced by $\mathrm{H} / \mathrm{R}$ insult in a dose-dependent manner ( $\mathrm{H} / \mathrm{R}$ vs. Pro $10, p=0.045$; $\mathrm{H} / \mathrm{R}$ vs. Pro $25, p<0.001 ; \mathrm{H} / \mathrm{R}$ vs. Pro $50, p<0.001 ; \mathrm{H} / \mathrm{R}$ vs. Pro 100 , $p<0.001)$. However, a peak increase in cell viability was observed with pretreatment of propofol at a dose of $50 \mu \mathrm{M}$, probably due to the saturation of ligand binding (Fig. 1).

\section{Propofol attenuates $\mathrm{LDH}$ release induced by $\mathrm{H} / \mathrm{R}$ injury in HK-2 cells}

We then evaluated the effects of propofol on $\mathrm{LDH}$ release in HK-2 cells. As shown in Fig. 2, H/R increased LDH release 3.8-fold over that in the control group, which was significantly attenuated by propofol in a dose-dependent manner. Maximal attenuation of LDH release (1.5-fold that of the control group) was observed at a dose of $50 \mu \mathrm{M}$ propofol. Interestingly, $100 \mu \mathrm{M}$ propofol seemed to increase LDH leakage, compared to $50 \mu \mathrm{M}$ propofol, although there was no statistical difference $(p>0.05)$. The possible cause was related to cytotoxicity from the high concentration of propofol (Fig. 2).

\section{Propofol pretreatment attenuates H/R induced cell apoptosis in HK-2 cells}

The effects of propofol pretreatment were also determined by detecting cell apoptosis using flow cytometry. As shown in Fig. 3, $\mathrm{H} / \mathrm{R}$ injury lead to a 3.5 -fold increase in cell apoptosis, compared to the control group $(p<0.001)$, and this alteration was reduced by propofol treatment (H/R vs. Pro $10, p=0.038 ; \mathrm{H} / \mathrm{R}$ vs. Pro 25 , $p=0.003 ; \mathrm{H} / \mathrm{R}$ vs. Pro $50, p<0.001 ; \mathrm{H} / \mathrm{R}$ vs. Pro $100, p<0.001)$. Consistently, peak reduction of cell apoptosis was obtained with treatment of $50 \mu \mathrm{M}$ propofol. Based on the above results, we chose $50 \mu \mathrm{M}$ propofol for subsequent experiments.

\section{Propofol pretreatment attenuates H/R-triggered cell apoptosis of HK-2 cells by inhibiting JNK activation} Given the essential role of JNK signal transduction pathway in cell apoptosis, we hypothesized that propofol would alleviate H/R-induced cell apoptosis in HK-2 cells by inhibiting JNK activation. To test this, we first ascertained the percentages of apoptotic cells in total cell cultures by measuring Annexin V-FITC and PI with flow cytometry (Fig. 4). As expected, we found that $\mathrm{H} / \mathrm{R}$



Fig. 1. Effects of propofol on hypoxia and reoxygenation (H/R)-induced cytotoxicity to HK-2 cells. Cell viability of the control group with neither $\mathrm{H}$ / R injury nor propofol pretreatment was used as a $100 \%$ benchmark. $H / R$ injury led to reduced cell viability (\#\# $p<0.01$ against the control group), and propofol pretreatment alleviated decreases in cell viability induced by $\mathrm{H}$ / $\mathrm{R}$ injury ( ${ }^{*} p<0.05$ and ${ }^{* *} p<0.01$ against the $\mathrm{H} / \mathrm{R}$ injury group). No significant difference was observed between pretreatments with $50 \mu \mathrm{M}$ and $100 \mu \mathrm{M}$ propofol (Pro 10, $10 \mu \mathrm{M}$; Pro 25, $25 \mu \mathrm{M}$; Pro 50, $50 \mu \mathrm{M}$; Pro 100, 100 $\mu \mathrm{M})$.

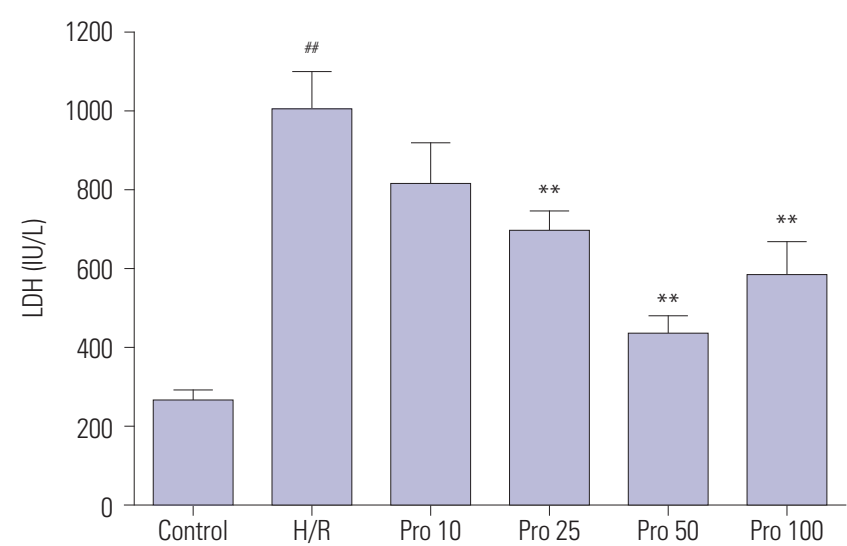

Fig. 2. Effects of propofol on LDH leakage in HK-2 cells. LDH levels were measured for the same control and experimental groups as in Fig. 1. Consistently, hypoxia and reoxygenation $(H / R)$ injury led to significantly higher levels of LDH ( ${ }^{\# \#} p<0.01$ against the control group), and propofol pretreatment alleviated increases in $\mathrm{LDH}$ caused by $\mathrm{H} / \mathrm{R}$ injury $\left(^{* *} p<0.01\right.$ against $H /$ R group).

injury induced cell apoptosis, while pretreatment with $50 \mu \mathrm{M}$ propofol alleviated the induced cell apoptosis $(p<0.01)$. Similar effects were also observed with pretreatment of $10 \mu \mathrm{M}$ SP600125 $(p<0.01)$. Interestingly, a combination of $50 \mu \mathrm{M}$ propofol and $10 \mu \mathrm{M}$ SP600125 offered stronger protection against cell apoptosis $(p<0.01)$, indicating potentially synergistic effects (combination of $50 \mu \mathrm{M}$ propofol and $10 \mu \mathrm{M}$ SP600125 versus $50 \mu \mathrm{M}$ propofol, $p<0.05$ ) We also examined the protein expressions levels of JNK, p-JNK, cleaved caspase-3, and Bcl-2 by Western blotting. As shown in Fig. 5, H/R injury resulted in elevated protein levels of p-JNK and cleaved caspase- 3 and decreased protein levels of Bcl-2 (all $p<0.01)$. All these changes were attenuated by pretreatment with $50 \mu \mathrm{M}$ propofol or $10 \mu \mathrm{M}$ SP600125 (all $p<0.05$ ), and more obvious attenuation was observed with a 


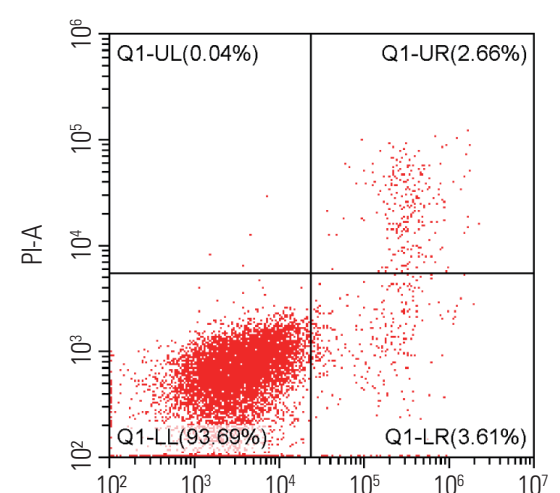

A

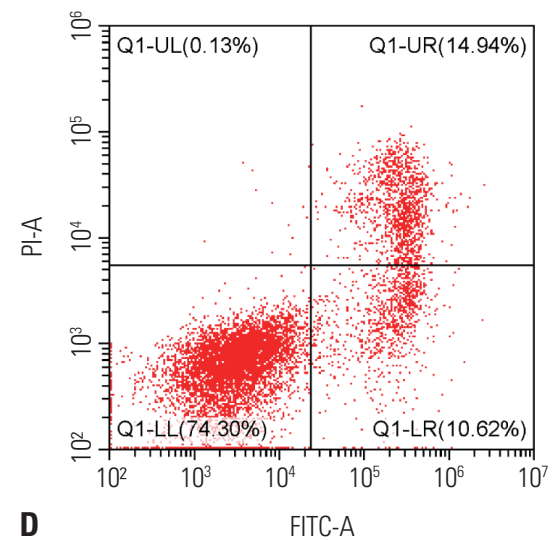

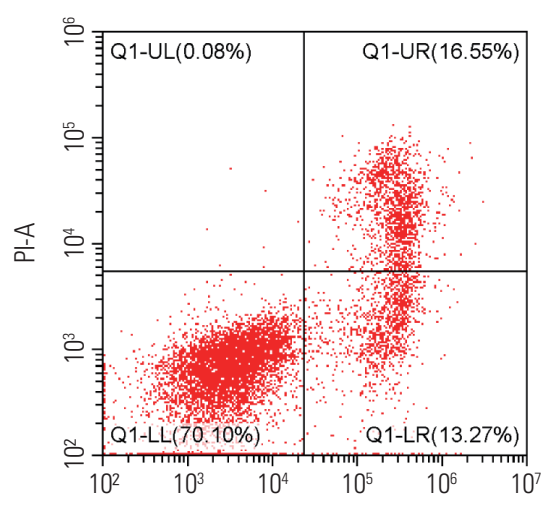

B

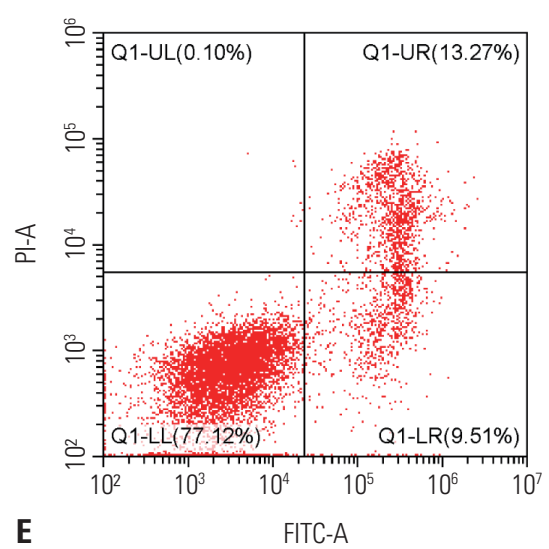

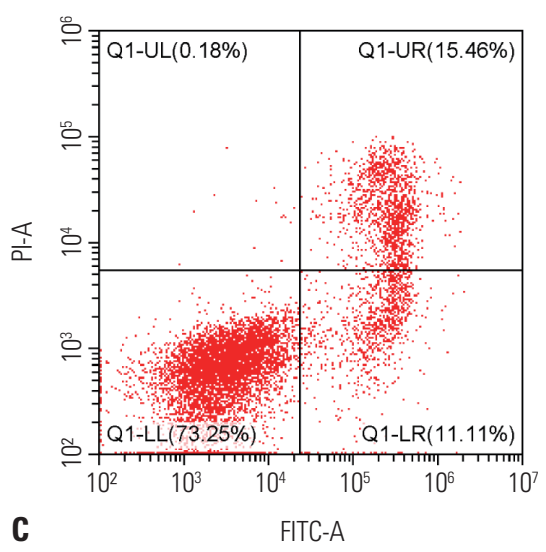

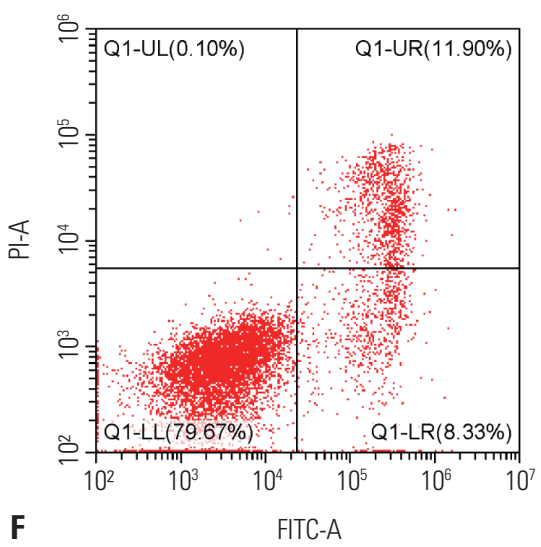

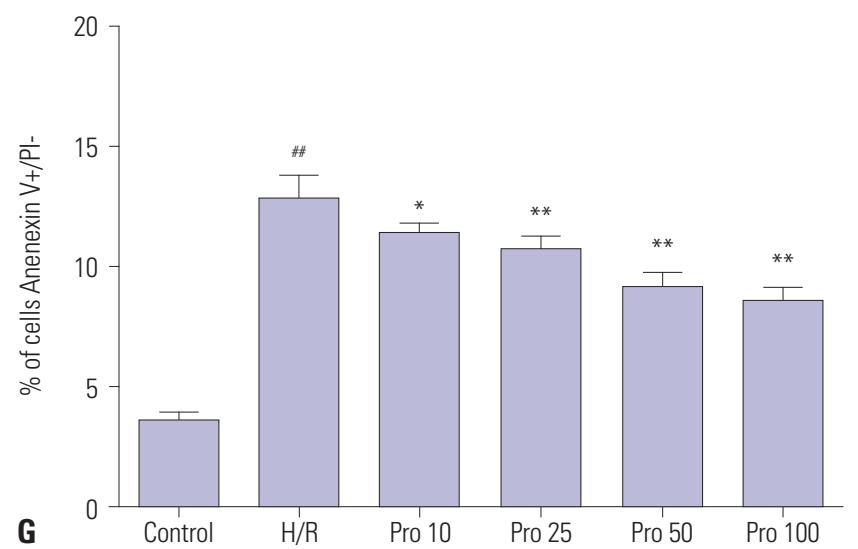

Fig. 3. Effects of propofol on apoptosis of HK-2 cells. Flow cytometry was conducted on the same control and experimental groups as in Fig. 1 (A: Control group; B: Hypoxia and reoxygenation (H/R) group; C: Pro 10 group; D: Pro 25 group; E: Pro 50 group; F: Pro 100 group). (G) Flow cytometric analysis for (A-F) was carried out as described in the Materials and Methods section. H/R injury increased the number of apoptotic cells $\left(^{\# \#} p<0.01\right.$ against the control group), and propofol pretreatment alleviated the observed increases ( ${ }^{*} p<0.05$ and ${ }^{* *} p<0.01$ against $\mathrm{H} / \mathrm{R}$ group).

combination thereof (all $p<0.01)$.

\section{Propofol alleviates H/R-induced autophagy}

To investigate whether propofol pretreatment attenuates the activation of H/R-induced autophagy, we also tested the levels of autophagy markers (LC3 and p62) via immunofluorescence and Western blot. As shown in Fig. 6A-C, the fluorescence intensity of anti-LC3II antibody was higher in the H/R group than that in the no-H/R control group $(p<0.01)$, suggesting elevated levels of autophagy. This alteration was partially reversed by $50 \mu \mathrm{M}$ propofol or $10 \mu \mathrm{M}$ SP600125 $(50 \mu \mathrm{M}$ propofol versus $\mathrm{H} / \mathrm{R}$, $p<0.01 ; 10 \mu \mathrm{M}$ SP600125 vs. H/R, $p<0.01)$. Like our findings with apoptosis, the combination of propofol and $\mathrm{SP} 600125$ provided greater alleviation against $\mathrm{H} / \mathrm{R}$-induced autophagy $(p<0.01)$. In a similar vein, changes in the fluorescence intensity of antip62 antibody also suggested that propofol alleviated H/R induced autophagy (all $p<0.05$ ). Western blots further supported our hypothesis. Propofol administration significantly inhibited 


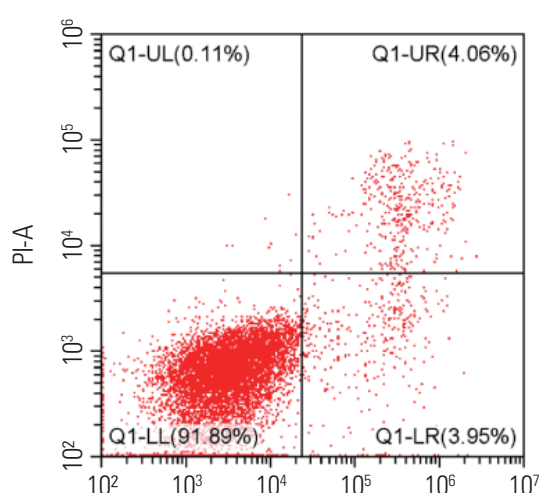

A

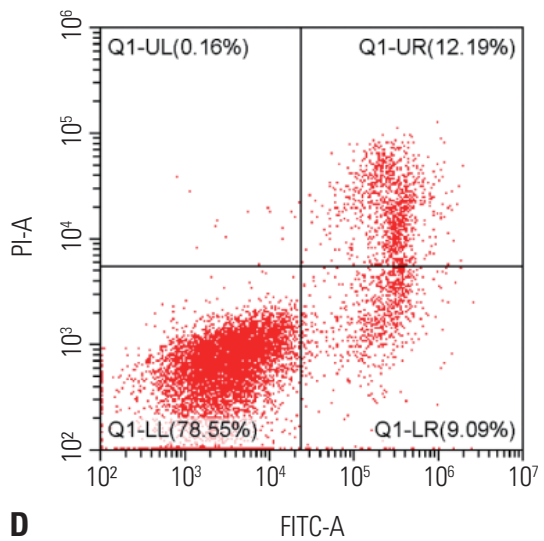

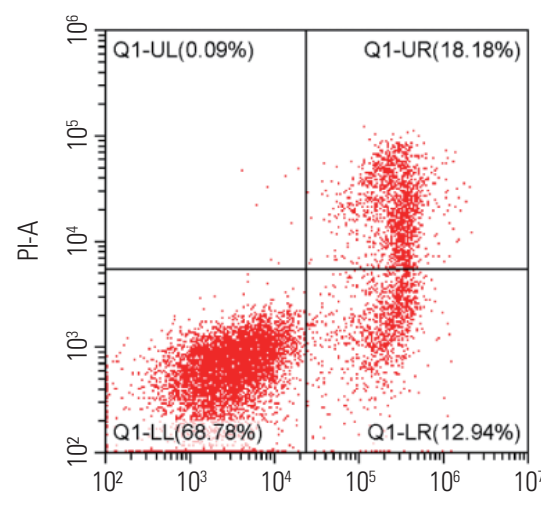

B

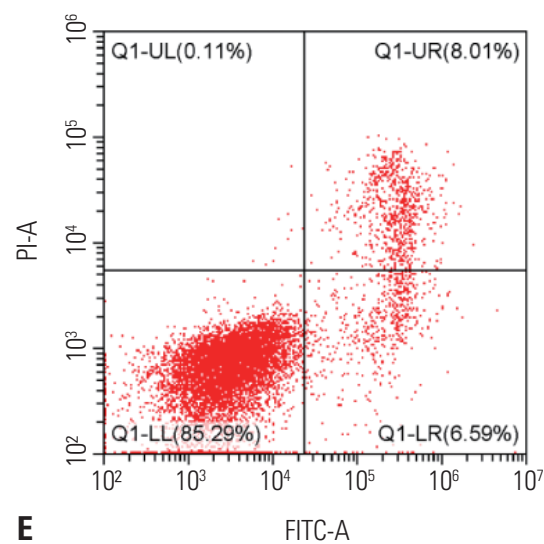

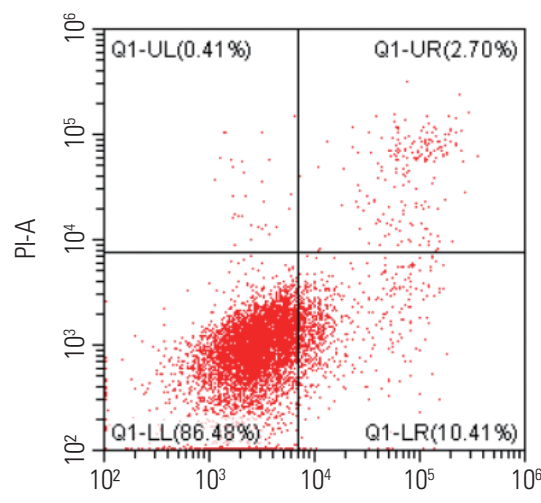

C

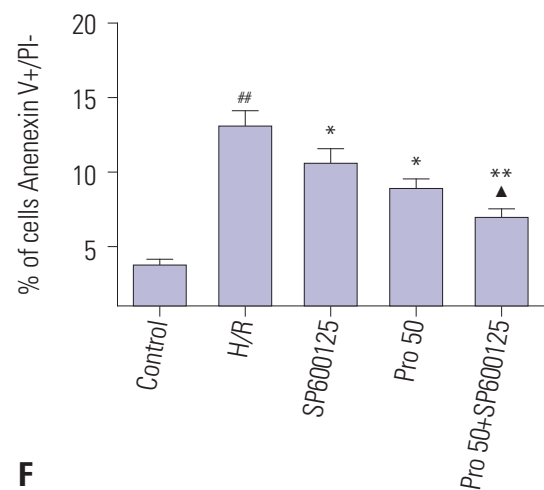

Fig. 4. Potential synergistic effect of propofol and SP600125 on alleviating cell apoptosis induced by hypoxia and reoxygenation (H/R) injury. (A-E) Representative figures of flow cytometry results for (A) the control group with neither $\mathrm{H} / \mathrm{R}$ injury nor propofol/SP600125 pretreatment, (B) the $\mathrm{H} / \mathrm{R}$ injury group with no pretreatment, (C) the SP600125 group pretreated with $10 \mu \mathrm{M} \mathrm{SP600125}$ prior to H/R injury, (D) the Pro 50 group pretreated with $50 \mu \mathrm{M}$ propofol prior to $\mathrm{H} / \mathrm{R}$ injury, and (E) the Pro 50+SP600125 group pretreated with a combination of $50 \mu \mathrm{M}$ propofol and $10 \mu \mathrm{M}$ SP600125 prior to H/R injury. (F) Flow cytometric analysis for (A-E) was carried out as described in the Materials and Methods section. $\mathrm{H} / \mathrm{R}$ injury increased cell apoptosis significantly ${ }^{\# \# ~} p<0.01$ against the control group), while a combination of propofol and SP600125 attenuated the increase in a potentially synergistic manner $\left({ }^{*} p<0.05\right.$ against $H / R$ group; ${ }^{* *} p<0.01$ against $\mathrm{H} / \mathrm{R}$ group; ${ }^{\mathbf{\Lambda}} p<0.05$ against Pro 50 group).

autophagy as demonstrated by decreased LC3II and increased P62 expression. Moreover, SP600125 strengthened the beneficial effect of propofol in HK2 cells exposed to hypoxia/reoxygenation injury (Fig. 6D-F; $p<0.05$ ).

\section{DISCUSSION}

To examine the effect of propofol on $\mathrm{H} / \mathrm{R}$ injury and its underlying mechanism, an in vitro model was established by subjecting HK-2 cells to H/R insult in this study. Decades of experimental results have shown that PTCs play a vital role in the histopathology of renal injury, especially in I/R impairment. ${ }^{11,12}$ After an acute ischemic insult, PTCs are the most sensitive cells to I/R injury, which is mainly due to physiological hypoxia in the medullary region and the high metabolic activity of PTCs. ${ }^{12}$ In recent years, numerous studies have used PTC models of H/R to investigate mechanisms of renal $\mathrm{I} / \mathrm{R}$ injury or to search for drugs that lower the risk of I/R injury. ${ }^{13,14}$ Several studies have shown that propofol is an excellent anti-inflammation and anti-oxi- dation agent, and may help to prevent and treat I/R injury, ${ }^{15,16}$ although the exact mechanisms remain unclear. We investigated the protective effects and underlying mechanisms of propofol against H/R-induced injury in HK-2 cells in the absence or presence of the JNK inhibitor SP600125. H/R injury was evaluated by measuring cell viability, LDH leakage, and apoptotic index. Studies have indicated that the clinically relevant concentrations of propofol were approximately $11-56 \mu \mathrm{M} \cdot{ }^{17,18}$ Thus, the concentration range of $10-100 \mu \mathrm{M}$ was used in this study, which made our results more clinically relevant. Our present study found a dose-dependent increase in cell viability, as well as dose-dependent decreases in LDH release and cell apoptosis, upon propofol treatment. All these indicate that propofol has a definite protective effect against H/R injury in HK-2 cells. Li, et al. ${ }^{16}$ reported that propofol prevented $\mathrm{H} / \mathrm{R}$ injury in rat renal tubular epithelial cell line (NRK-52E cells), similar to our results. Compared with $50 \mu \mathrm{M}$ of propofol, $100 \mu \mathrm{M}$ offered no extra protection against $\mathrm{H} / \mathrm{R}$ injury.

JNK is involved in cell apoptosis; however, it also acts synergistically with JAK/STAT, NF-KB, and other signaling molecules 


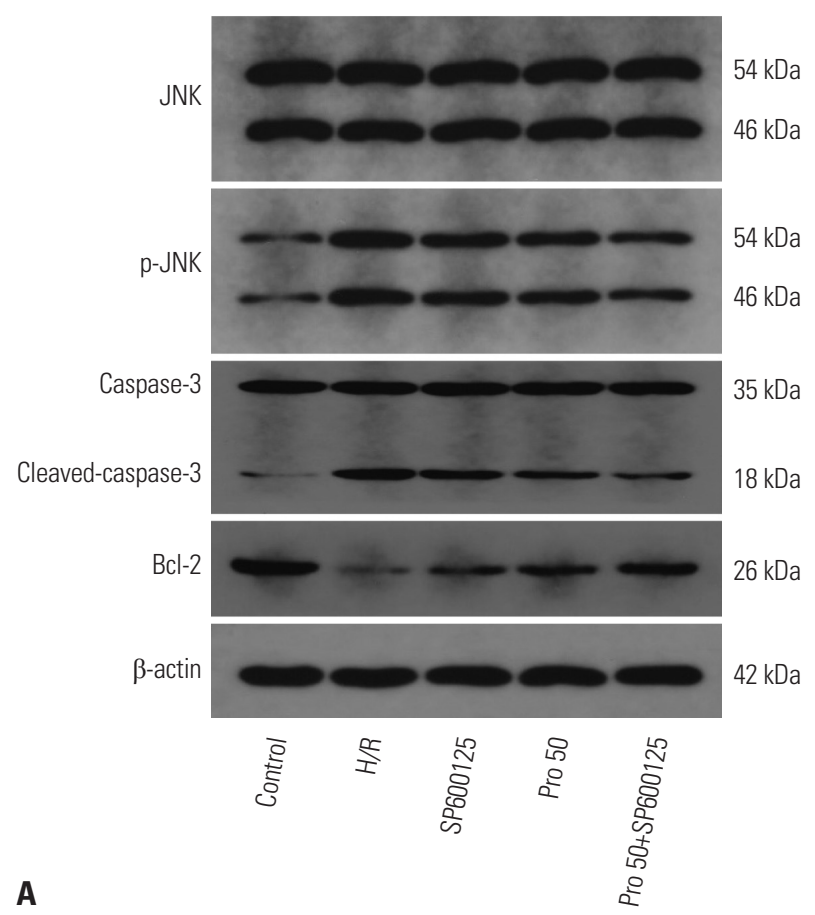

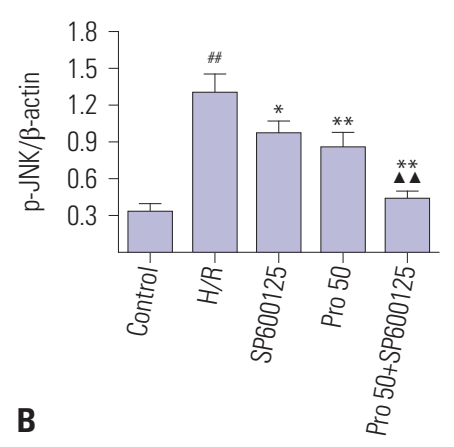
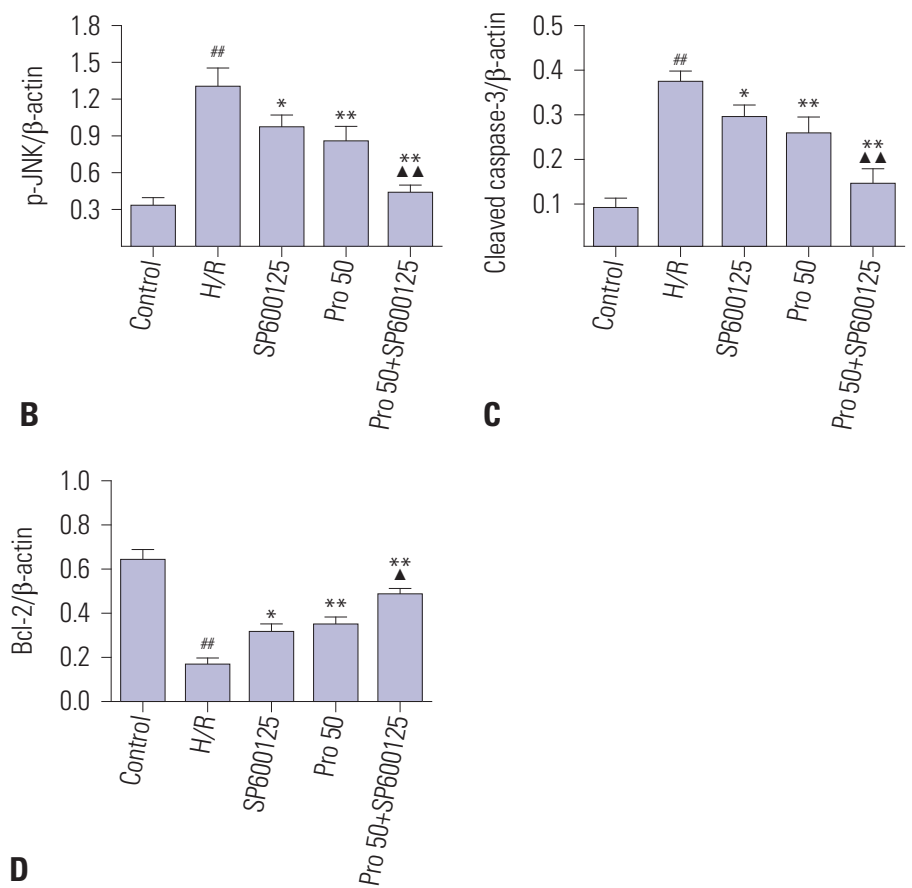

Fig. 5. Effects of propofol and SP600125 on the protein expression levels of JNK, p-JNK, caspase-3, cleaved-caspase-3, and Bcl-2 in HK-2 cells. Western blots were performed for the same control and experimental groups as in Fig. 4 to measure expression levels of the above-mentioned proteins. (A) Raw data. (B-D) Hypoxia and reoxygenation $(H / R)$ injury increased the protein expression levels of p-JNK and cleaved-caspase-3, and decreased those of Bcl-2. Both SP600125 and propofol attenuated the changes in expression levels, and a combination of both alleviated the changes further $\left({ }^{\# \#} p<0.01\right.$ against the control group; ${ }^{*} p<0.05$ and ${ }^{* *} p<0.01$ against the $\mathrm{H} / \mathrm{R}$ group; ${ }^{\mathbf{\wedge}} p<0.05$ and ${ }^{\mathbf{\wedge}} \boldsymbol{\Delta} p<0.01$ against the Pro 50 group).

to promote cancer cell survival. ${ }^{19}$ Studies have reported that JNK activation after $\mathrm{H} / \mathrm{R}$ is excessively increased in human brain vascular smooth muscle cells, ${ }^{20} \mathrm{H} 9 \mathrm{c} 2$ cardiomyocytes, ${ }^{21}$ and HK-2 cells. ${ }^{22}$ In the present study, we selected the specific JNK inhibitor SP600125 to ascertain the effect of propofol against H/R injury. Our results showed that propofol significantly attenuates H/R-induced cell apoptosis and JNK activation. Similar effects were shown by treatment with JNK inhibitor SP600125. These results indicated that the protective effects of propofol against $\mathrm{H} / \mathrm{R}$ injury may be related to JNK inhibition. An interesting finding in our study was that the beneficial effects of the combined use of propofol and SP600125 were more obvious than use of only one them. These indicate their combination has better superimposed protective effects.

Autophagy is a critical mechanism for cellular homeostasis and survival in response to renal I/R injury. However, it is believed to be a double-edged sword in cell injury. On the one hand, physiologic autophagy is involved in degradation of harmful proteins and damaged organelles to prevent the accumulation of harmful substances, which limits the transmission of harmful signaling. On the other hand, excessive autophagy will cause irreversible injury and transform cells to autophagic cell death. To ascertain the role of autophagy in propofol-induced protection, HK-2 cells were pretreated with propofol in the absence or presence of SP600125 prior to H/R injury. We showed that autophagy was significantly increased after $\mathrm{H} / \mathrm{R}$ as indicated by a significant increase in LC3II protein levels and a decrease in p62 protein levels, and propofol attenuated these changes, which were further alleviated by a combination of propofol and SP600125. Likewise, Xie, et al. ${ }^{23}$ reported that autophagosomes and autolysosomes were significant increased at $2 \mathrm{~h}$ after reoxygenation in HK-2 cells. According to previous reports, inhibition of the JNK pathway by SP600125 could block autophagy activation in cancer cells. ${ }^{24}$ We showed here that SP600125 attenuated H/R-triggered autophagy activation. Although the exact mechanism by which propofol-inhibited autophagy protects from H/R injury is unclear, our results showed that it is likely that propofol inhibits excessive autophagy during the injury partly through JNK pathway. It was confirmed that some situations, including overdigestion of cytoplasmic contents due to excessive autophagy, might result in autophagic cell death. ${ }^{25}$ Recent studies have reported that propofol antagonizes H/R-triggered neuronal injury via inhibition of autophagy. ${ }^{7}$ Other studies have stated that propofol postconditioning protects $\mathrm{H} 9 \mathrm{c} 2$ cells from H/R injury by inducing autophagy via JNK pathway. ${ }^{26}$ This discrepancy could result from differences in the degree of autophagy activation or test subjects.

More should be done in future studies to verify the protective effect of propofol on H/R injury particularly with in vivo assay. Also, reoxygenation time may affect the expression levels of related proteins after H/R injury, ${ }^{14}$ and in this study, we harvested the cells only at $2 \mathrm{~h}$ after reoxygenation due to limited resources. Therefore, it would be informative to measure the effects of propofol against H/R injury at different reoxygenation times. 
Despite the limitations, our study has demonstrated unambiguously that propofol helps to protect HK-2 cells against H/R injury by alleviating apoptosis and autophagy, and suggests that the protective effects are derived from its inhibition of JNK activation.
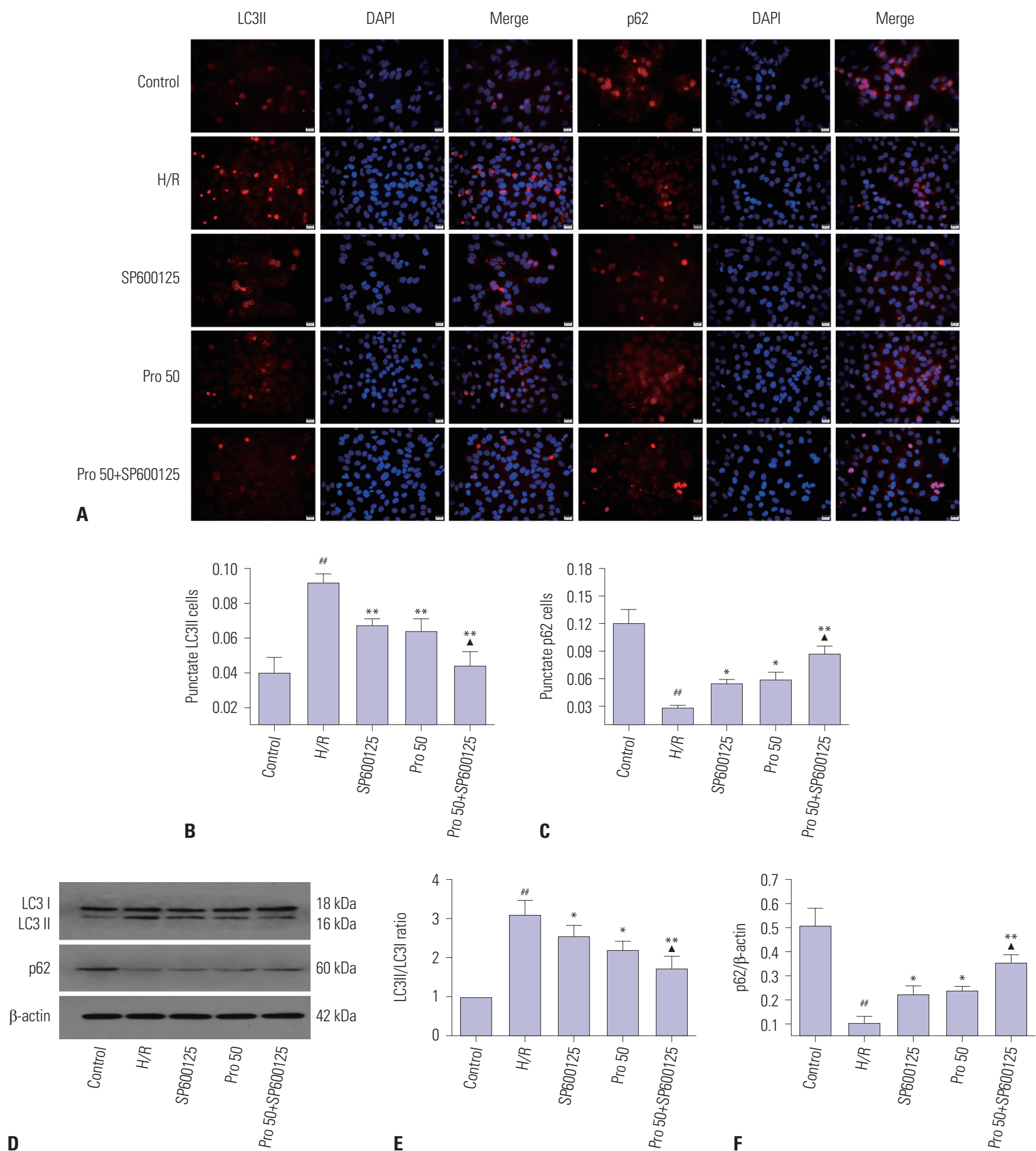

Fig. 6. Effects of propofol and SP600125 on autophagy induced by hypoxia and reoxygenation (H/R) injury. The same control and experimental groups as in Fig. 4 were subjected to fluorescence microscopy (A-C) and Western blots (D-F) to measure the protein expression levels of LC3II and p62 (autophagy markers). Both techniques showed that H/R injury increased the protein expression levels of LC3II and decreased those of p62. Both SP600125 and propofol attenuated the changes, and their alleviating effects were stronger when applied together ${ }^{\# \#} p<0.01$ against the control group; ${ }^{*} p<0.05$ and ${ }^{* *} p<0.01$ against the $\mathrm{H} / \mathrm{R}$ group; ${ }^{\boldsymbol{}} p<0.05$ against the Pro 50 group). 


\section{ACKNOWLEDGEMENTS}

We thank the Central Laboratory, Wuhan University Renmin Hospital for providing facilities.

This study was supported by the China Scholarship Council (No. 201706275133), the Natural Science Foundation of Hubei Province (No. 2019CFC853), and the Basic Scientific Research Foundation of Central Universities (No. 2042017kf0144).

\section{AUTHOR CONTRIBUTIONS}

Conceptualization: Huaxin Wang. Data curation: Huaxin Wang and Xuan Peng. Formal analysis: Yeda Xiao and Xuan Peng. Funding acquisition: Huaxin Wang. Investigation: Zhuo Wang. Methodology: Yayi Huang and Xuan Peng. Project administration: Huaxin Wang and Liying Zhan. Resources: Huaxin Wang. Software: Yeda Xiao. Supervision: Liying Zhan. Validation: Huaxin Wang and Liying Zhan. Visualization: Yayi Huang and Zhuo Wang. Writing_original draft: Huaxin Wang, Yayi Huang, and Zhuo Wang. Writing—review \& editing: Huaxin Wang and Liying Zhan.

\section{ORCID iDs}

$\begin{array}{ll}\text { Huaxin Wang } & \text { https://orcid.org/0000-0003-0110-0647 } \\ \text { Xuan Peng } & \text { https://orcid.org/0000-0001-5505-3837 } \\ \text { Yayi Huang } & \text { https://orcid.org/0000-0002-3357-7864 } \\ \text { Yeda Xiao } & \text { https://orcid.org/0000-0001-7340-6990 } \\ \text { Zhuo Wang } & \text { https://orcid.org/0000-0001-7261-0818 } \\ \text { Liying Zhan } & \text { https://orcid.org/0000-0002-7368-9932 }\end{array}$

\section{REFERENCES}

1. Saat TC, van den Akker EK, IJzermans JN, Dor FJ, de Bruin RW. Improving the outcome of kidney transplantation by ameliorating renal ischemia reperfusion injury: lost in translation? J Transl Med 2016;14:20.

2. Chouchani ET, Pell VR, James AM, Work LM, Saeb-Parsy K, Frezza C, et al. A unifying mechanism for mitochondrial superoxide production during ischemia-reperfusion injury. Cell Metab 2016;23: 254-63.

3. Hamzawy M, Gouda SAA, Rashed L, Morcos MA, Shoukry H, Sharawy N. 22-oxacalcitriol prevents acute kidney injury via inhibition of apoptosis and enhancement of autophagy. Clin Exp Nephrol 2019;23:43-55.

4. Smith SF, Hosgood SA, Nicholson ML. Ischemia-reperfusion injury in renal transplantation: 3 key signaling pathways in tubular epithelial cells. Kidney Int 2019;95:50-6.

5. Su M, Ren S, Zhong W, Han X. Impact of propofol on renal ischemia/reperfusion endoplasmic reticulum stress. Acta Cir Bras 2017;32:533-9.

6. Decuypere JP, Pirenne J, Jochmans I. Autophagy in renal ischemia-reperfusion injury: friend or foe? Am J Transplant 2014;14: 1464-5.

7. Sun B, Ou H, Ren F, Huan Y, Zhong T, Gao M, et al. Propofol inhibited autophagy through $\mathrm{Ca}(2+) / \mathrm{CaMKK} \beta / \mathrm{AMPK} / \mathrm{mTOR}$ pathway in OGD/R-induced neuron injury. Mol Med 2018;24:58.

8. Zhu Y, Li S, Liu J, Wen Q, Yu J, Yu L, et al. Role of JNK signaling path- way in dexmedetomidine post-conditioning-induced reduction of the inflammatory response and autophagy effect of focal cerebral ischemia reperfusion injury in rats. Inflammation 2019 Aug 24 [Epub]. Available at: https://doi.org/10.1007/s10753-019-01082-2.

9. Jiang M, Fan J, Qu X, Li S, Nilsson SK, Sun YBY, et al. Combined blockade of Smad3 and JNK pathways ameliorates progressive fibrosis in folic acid nephropathy. Front Pharmacol 2019;10:880.

10. Ibrahim YF, Moussa RA, Bayoumi AMA, Ahmed AF. Tocilizumab attenuates acute lung and kidney injuries and improves survival in a rat model of sepsis via down-regulation of NF- $\mathrm{KB} / \mathrm{JNK}$ : a possible role of P-glycoprotein. Inflammopharmacology 2019 Aug 22 [Epub]. Available at: https://doi.org/10.1007/s10787-019-00628-y.

11. Moonen L, D'Haese PC, Vervaet BA. Epithelial cell cycle behaviour in the injured kidney. Int J Mol Sci 2018;19. pii: E2038.

12. Granger DN, Kvietys PR. Reperfusion injury and reactive oxygen species: the evolution of a concept. Redox Biol 2015;6:524-51.

13. Ha SD, Solomon O, Akbari M, Sener A, Kim SO. Histone deacetylase 8 protects human proximal tubular epithelial cells from hypoxia-mimetic cobalt- and hypoxia/reoxygenation-induced mitochondrial fission and cytotoxicity. Sci Rep 2018;8:11332.

14. Lu J, Yi Y, Pan R, Zhang C, Han H, Chen J, et al. Berberine protects HK-2 cells from hypoxia/reoxygenation induced apoptosis via inhibiting SPHK1 expression. J Nat Med 2018;72:390-8.

15. Yoo YC, Yoo KJ, Lim BJ, Jun JH, Shim JK, Kwak YL. Propofol attenuates renal ischemia-reperfusion injury aggravated by hyperglycemia. J Surg Res 2013;183:783-91.

16. Li Y, Zhong D, Lei L, Jia Y, Zhou H, Yang B. Propofol prevents renal ischemia-reperfusion injury via inhibiting the oxidative stress pathways. Cell Physiol Biochem 2015;37:14-26.

17. Hiraoka H, Yamamoto K, Miyoshi S, Morita T, Nakamura K, Kadoi $\mathrm{Y}$, et al. Kidneys contribute to the extrahepatic clearance of propofol in humans, but not lungs and brain. Br J Clin Pharmacol 2005; 60:176-82.

18. Kanto J, Gepts E. Pharmacokinetic implications for the clinical use of propofol. Clin Pharmacokinet 1989;17:308-26.

19. Wu Q, Wu W, Fu B, Shi L, Wang X, Kuca K. JNK signaling in cancer cell survival. Med Res Rev 2019;39:2082-104.

20. Zhang J, Xia Y, Xu Z, Deng X. Propofol suppressed hypoxia/reoxygenation-induced apoptosis in HBVSMC by regulation of the expression of Bcl-2, Bax, Caspase3, Kir6.1, and p-JNK. Oxid Med Cell Longev 2016;2016:1518738.

21. Wang D, Chen T, Liu F. Betulinic acid alleviates myocardial hypoxia/reoxygenation injury via inducing Nrf2/HO-1 and inhibiting p38 and JNK pathways. Eur J Pharmacol 2018;838:53-9.

22. Yan R, Zhang L, Xia N, Liu Q, Sun H, Guo H. Knockdown of augmenter of liver regeneration in HK-2 cells inhibits inflammation response via the mitogen-activated protein kinase signaling pathway. Inflamm Res 2015;64:453-62.

23. Xie Y, Xiao J, Fu C, Zhang Z, Ye Z, Zhang X. Ischemic preconditioning promotes autophagy and alleviates renal ischemia/reperfusion injury. Biomed Res Int 2018;2018:8353987.

24. Ren H, Fu K, Mu C, Li B, Wang D, Wang G. DJ-1, a cancer and Parkinson's disease associated protein, regulates autophagy through JNK pathway in cancer cells. Cancer Lett 2010;297:101-8.

25. Lin CW, Lo S, Perng DS, Wu DB, Lee PH, Chang YF, et al. Complete activation of autophagic process attenuates liver injury and improves survival in septic mice. Shock 2014;41:241-9.

26. Li H, Zhang X, Tan J, Sun L, Xu LH, Jiang YG, et al. Propofol postconditioning protects $\mathrm{H} 9 \mathrm{c} 2$ cells from hypoxia/reoxygenation injury by inducing autophagy via the SAPK/JNK pathway. Mol Med Rep 2018;17:4573-80. 